\title{
Cluster Analyzing Profile Data Confounded with Interrater Differences: A Comparison of Profile Association Measures
}

\author{
Robert M. Hamer \\ The University of lowa \\ J.W. Cunningham \\ North Carolina State University
}

Seven association measures were compared for their effectiveness in relating and clustering data profiles confounded with interrater differences. Among these association indices were three distance measures, two measures of angular separation, and two measures of profile overlap. The objects of analysis were 50 jobs that had been rated by different analysts on the items comprising the Occupation Analysis Inventory (OAI). Factor scores based on the OAI job ratings provided the profile data. Each of the seven association measures was applied to all pairwise combinations of factor-score profiles in the job sample, and the seven resultant $50 \times 50$ job proximity matrices were each subjected to hierarchical cluster analysis. The job proximity matrices and cluster structures based on the different association measures were then compared, respectively, with a criterion proximity matrix and a criterion cluster structure. In relation to these two criteria, the angular measures (product-moment correlation and cosine) performed better than the distance and overlap measures. The results demonstrate the importance of the choice of a profile association measure in cluster analysis. The researcher should be especially cautious when clustering entities that have been rated by different judges. Under such circumstances, it might be advisable to cluster analyze a data set using more than one association measure and then to compare the alternative solutions for clarity and stability.

APPLIED PSYCHOLOGICAL MEASUREMENT

Vol. 5, No. 1, Winter 1981, pp. 63-72

(C) Copyright 1981 Applied Psychological Measurement Inc.
Many taxonomic studies in psychology involve clustering objects of analysis (e.g., individuals or stimuli) according to their data profiles on selected variables. The general objective of such research is to derive meaningful cluster structures that tend to minimize within-group profile differences or maximize within-group similarities.

In undertaking an effort of this kind, the investigator must make decisions related to such basic issues as (1) the selection of relevant profile variables; (2) the appropriate transformation of profile data; (3) the appropriate measure of profile association; (4) the appropriate clustering criterion; and (5) the choice of an implementing algorithm and computer program (Anderberg, 1973, chap. 2). At each decision point the researcher is confronted with a variety of competing alternatives, from which the choice to be made should be based upon the characteristics and requirements of his/her particular problem. Unfortunately, sufficient bases for these decisions often cannot be found in the research literature. Thus Borgen and Weiss (1971) have noted "when cluster analysis is appraised as a methodology, the number of unanswered questions surpasses the number of present answers ... insufficient comparative data are available to say with certainty that this method is best for this kind of grouping problem" (p. 590). Almost 10 years later, this observation still accurately 
describes a troublesome situation in classificatory research.

The study reported here stemmed from a need to resolve one of the above-mentioned clustering issues within a particular research setting. Prior to this study Riccobono, Cunningham, and Boese (1975) had applied cluster analysis to the data profiles of 814 jobs. The profile variables in this analysis were 22 second-order factors derived from the Occupation Analysis Inventory, an instrument containing 615 "work elements" (items) on which the jobs were rated (Cunningham, Tuttle, Floyd, \& Bates, 1974; Riccobono \& Cunningham, 1974). Squared Euclidean distances $\left(D^{2}\right)$ were computed for all pairwise combinations of factor score profiles, and the resultant job proximity matrix was subjected to a hierarchical cluster analysis (Ward, 1961, 1963). This analysis, however, failed to produce a meaningful job cluster structure.

In a postmortem of their study Riccobono et al. (1975) identified several factors that might have attenuated the results, foremost of which was the $D^{2}$ measure of profile association. The $D^{2}$ index and its square root are often used in cluster analysis because they are mathematically tractable and are sensitive to the three major information components in a score profile: level, dispersion, and shape (Cronbach \& Gleser, 1953; Nunnally, 1978; Osgood \& Suci, 1952). In retrospect, however, the investigators reasoned that the level and dispersion components in their profile data may have been contaminated by the individual judgment tendencies of the 18 different analysts who rated the jobs. Since each job was rated by only one of those analysts, any individual differences in the analysts' adaptation levels and rating dispersions (as well as other interrater differences) would have been confounded with legitimate profile differences. Large interrater differences could therefore be expected to distort the $D^{2}$ measure and, hence, the job cluster structure. Under such circumstances a job proximity measure that disregarded profile level and dispersion might have been appropriate.
Another potential difficulty with the $D^{2}$ measure lay in the skewness of some of the factorscore distributions, where disproportionatc numbers of jobs had low scores. In this connection, it was argued that a shared irrelevance of such factors between jobs (i.e., paired low scores), compared to shared relevance on other factors, might have contributed inordinately to some of the job proximities. If this were, in fact, the case, a profile association measure that attached more weight to paired high scores thar paired low scores would have been preferable to $D^{2}$.

Based on the foregoing considerations, it was decided that an evaluative analysis of several proximity measures should be performed preparatory to any further job-clustering efforts involving the Occupation Analysis Inventory (OAI). The present study was designed for that purpose.

\section{Method}

The approach to this problem involved (1) applying several selected proximity measures to all pairwise combinations of OAI factor-score profiles in a job sample, (2) evaluating the resultant job proximity matrices against a criterion proximity matrix, (3) cluster analyzing each of the job proximity matrices, and (4) evaluating the resultant job cluster structures against a criterion cluster structure.

\section{Criteria and Job Sample}

Criterion cluster structure. The criterion cluster structure consisted of six sets of jobs representing six Worker Trait Groups (WTGs) selected from the Worker Traits Arrangement of the Dictionary of Occupational Titles (DOT); U. S. Department of Labor, 1965). The six WTGs were chosen to represent a range of occupations, while at the same time providing a test of the OAI's power of resolution. Thus, two pairs of selected WTGs contained groups that were somewhat similar to each other but dis- 
similar to those in the other pair, and the two remaining WTGs were dissimilar to each other and to all other groups in the set. The titles of the selected WTGs appear in Table 1.

Job sample. Fifty jobs were selected to represent the six WTGs in the present study. Over several years of previous research, a cumulative sample of 1,414 jobs had been rated on the OAI by various analysts. In most cases only one analyst rated each job, though in some instances a job was rated by two analysts whose ratings were averaged. The 50 jobs included in this study were chosen from the total sample based on comparisons of their titles and $D O T$ codes to those of the WTGs' constituent occupations as listed in the DOT. The jobs' OAI ratings were used to generate profile data for the study. Table 1 identifies the jobs selected for each WTG.

Criterion proximity matrix. The criterion proximity matrix contained job similarity estimates obtained from judges' ratings. Each of nine judges, all psychology graduate students, was given written descriptions (U. S. Employment Service job schedules) of the 50 selected jobs, which he/she used in making pairwise job similarity ratings. These ratings were performed on a 9-point scale ranging from " 1 -extremely similar" to "9-extremely dissimilar." A $50 \times 50$ job proximity matrix was obtained for each judge with the aid of Baker and Young's (1974) Interactive Scaling with Individual Subjects (ISIS) system. This computerized procedure samples a judge's pairwise similarity ratings and, using those ratings as a model, estimates the remaining rating values needed to produce a complete, symmetric judgment matrix.

An interrater agreement analysis was carried out to determine if any judge's similarity ratings deviated excessively from those of the other judges. This analysis involved correlating each judge's pairwise job similarity ratings with the mean pairwise ratings of the remaining judges. The two judges with the lowest correlation coefficients $(-.12$ and .14) were eliminated from subsequent analyses, leaving seven judges whose coefficients ranged from .38 to .93 . Of these, six judges' coefficients exceeded .67 and four exceeded .88 .

The reliability of the remaining seven judges' combined ratings was then estimated through an analysis-of-variance procedure in which judges were categories of the "treatment" variable, job pairs were cases on which repeated measures were taken, and pairwise similarity ratings were the measures (cell entries; Winer, 1971, chap. 4). From this procedure the reliability coefficient for the mean pairwise similarity ratings of the seven judges was .84 (unadjusted for differences in the judges' anchor points). These pairwise means comprised the entries in a $50 \times 50$ criterion proximity matrix.

\section{Profile Variables}

The profile variables were factors derived from the OAI. In a preceding study (Boese \& Cunningham, 1976) eight separate a priori sections of OAI work elements (items) had each been subjected to a principal axis factor analysis based on the OAI ratings of 1,414 jobs. The eight separate analyses produced a total of 132 first-order factors representing basic types of work activities and conditions. (Although the factors were rotated orthogonally within each of the eight OAI sections, orthogonality was not maintained among factors across the different sections.) Of those factors, 102 were selected for use in the present study.

The factor scores for the $\mathbf{5 0}$ jobs in this study were incomplete estimates based on salient loadings (cf. Horn, 1965). Specifically, a factor score consisted of the mean cross-product between the factor's salient loadings (transformed to Fisher's $z$ 's) and the job's corresponding work-element ratings. Since incomplete estimates violate the orthogonality of an original factor structure, there were correlations among the estimated factor scores within, as well as across, the eight $\mathrm{OAI}$ sections.

\section{Job Proximity Measures}

Based on previously mentioned considerations, seven proximity measures were selected 
Table I

Jobs Selected to Represent Six worker Trait Groups (W'l'G's)

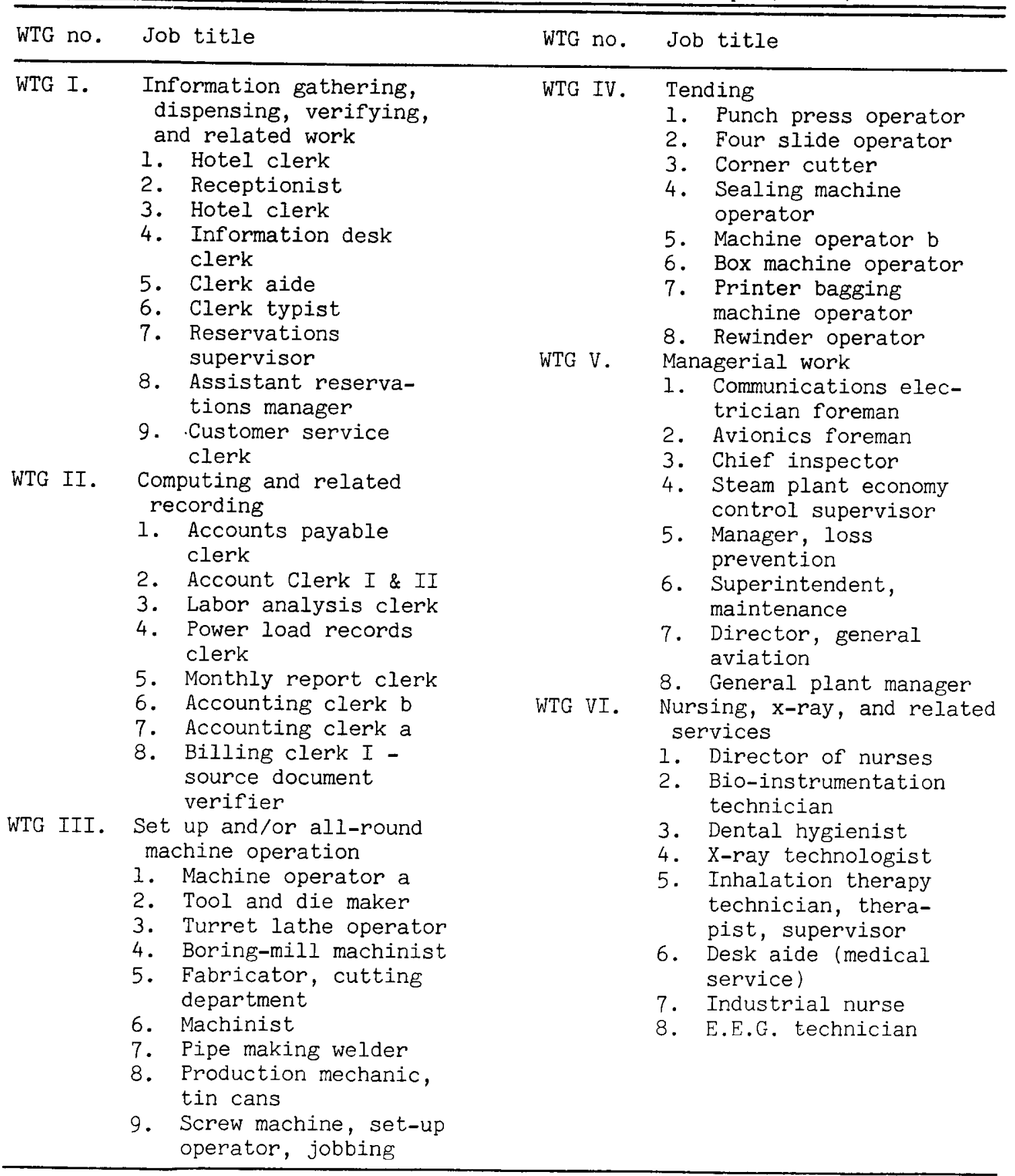


for comparative analysis. The selection included three distance measures, two angular measures, and two measures of profile "overlap." Table 2 identifies these seven measures and presents their formulas.

Distance measures. As noted, the Euclidean distance $(D)$ and its square are sensitive to profile level, dispersion, and shape. For that reason and because $D^{2}$ had been used by Riccobono et al. (1975), both measures were included in the present study. In addition, a third distance measure, average nonzero distance (AND), was devised especially for this study. It was hoped that the AND index, by disregarding paired zero scores between two profiles, would reduce the influence of mutually irrelevant variables upon measured job proximity. However, AND as well as $D$ and $D^{2}$ may be susceptible to considerable distortion from interrater differences.

Angular measures. The two angular measures of job proximity were the cosine and the product-moment correlation. The cosine of the angle between two score vectors assumes ratio scaling. Though sensitive primarily to similarities and differences in profile shape, this index is affected to some extent by the level and dispersion components. The product-moment correlation (the cosine of the angle between two centered score vectors) assumes interval scaling and is sensitive only to profile shape (cf. Anderberg, 1973, chap. 4; Nunnally, 1978, chap. 12). Thus, differences in two job analysts' adaptation levels and rating dispersions should not affect the correlation between their job-rating (factorscore) profiles, provided such differences do not influence the proportionality of the intraprofile score intervals. The cosine, on the other hand, would be affected not only by rater distortions of intraprofile intervals but also by rater differences in the intraprofile proportionality of scores. However, since both angular measures reflect primarily the rank-order correspondence between two score profiles, neither measure should be distorted greatly by individual rating tendencies that do not disturb the ordinal characteristics of the profiles (Nunnally, 1978, chap.
1). Accordingly, it was predicted that the angular measures would prove more effective than the distance measures in the present study.

Measures of overlap. The U. S. Air Force and other military services use a job proximity measure called percent time overlap (PO), an index based on jobs' time-spent ratings on common tasks (Archer, 1966; Morsh, 1965). In the computation of this index, each job's individual task ratings are converted to percentages of that job's total time-spent rating on all tasks. The PO between two jobs is the sum of the smaller values in their paired converted task ratings, i.e., their total PO on tasks. This procedure standardizes profile level.

Although the OAI factor scores are based on significance and extent rather than time-spent ratings, PO was selected as a potentially effective measure of job proximity in this study. Like the cosine, PO assumes ratio scaling, that is, it is invariant only to multiplicative changes in score profiles. Although PO is more sensitive than the cosine and correlation to other monotonic changes in score profiles, this index is less sensitive than the distance measures to such transformations. Hence, PO should be more susceptible than the angular measures to distortion by interrater differences but less susceptible than the distance measures.

$A$ variation of the $\mathrm{PO}$ index, absolute overlap (AO), was the final job proximity measure selected for this study. The AO between two jobs is the sum of the smaller values in their paired factor scores, i.e., the total overlap in their factor scores. Since AO does not standardize profile level, this index should be more susceptible than PO to distortion by interrater differences.

\section{Analyses}

The analyses were designed to evaluate the seven job proximity measures against two previously described standards: the criterion proximity matrix and the criterion cluster structure.

Proximity matrices. Each of the selected proximity measures was applied to all pairwise 
Table 2

Formulas for the Seven Selected Proximity Measures

Proximity Measure Formula

EUCLIDEAN DISTANCE

SQUARED EUCLIDEAN DISTANCE

AVERAGE NON-ZERO DISTANCE

COSINE

PRODUCT-MOMENT CORRELATION

PERCENT OVERLAP

ABSOLUTE OVERLAP

$$
\begin{aligned}
D & =\left[\sum_{i=1}^{n}\left(x_{i}-y_{i}\right)^{2}\right]^{1 / 2} \\
D^{2} & =\sum_{i=1}^{n}\left(x_{i}-y_{i}\right)^{2}
\end{aligned}
$$$$
A N D=\frac{\left[\sum_{i=1}^{n}\left(x_{i}-y_{i}\right)^{2}\right]^{1 / 2}}{n-k}
$$$$
\operatorname{Cos}=\frac{\sum_{i=1}^{n} x_{i} y_{i}}{\left[\left(\sum x_{i}^{2}\right)\left(\sum y_{i}^{2}\right)\right] 1 / 2}
$$$$
r=\frac{\sum_{i=1}^{n}\left(x_{i}-\bar{x}\right)\left(y_{i}-\bar{y}\right)}{\left\{\left[\sum_{i=1}^{n}\left(x_{i}-\bar{x}\right)^{2}\right]\left[\sum_{i=1}^{n}\left(y_{i}-\bar{y}\right)^{2}\right]\right\}^{1 / 2}}
$$$$
\text { PO }=\sum_{i=1}^{n} \operatorname{Minimum}\left(x_{i}^{*}, y_{i}^{*}\right)
$$$$
\Delta O=\sum_{i=1}^{n} \operatorname{Minimum}\left(x_{i}, y_{i}\right)
$$

Note: The symbols in the above formulas are defined as follows:

$X_{\mathbf{i}}$ and $Y_{\mathbf{i}}$ represent the $\boldsymbol{i}$ th elements in the data vectors of Jobs $X$ and $Y$, respectively.

$\bar{X}$ and $\bar{Y}$ represent the means of the elements in the data vectors of Jobs $X$ and $Y$, respectively.

$\mathbf{n}=$ the number of elements in the data vector of Job $X$ or Job $Y$.

$\mathbf{k}=$ the number of element pairs in which $X_{i}=Y_{1}=0$.

$x_{i}^{*}=x_{i} / \sum_{i=1}^{n} X_{i}$ and $Y_{i}^{*}=Y_{i} / \sum_{i=1}^{n} Y_{i}$ 
combinations of factor-score profiles in the job sample. The seven resultant $50 \times 50$ job proximity matrices were then intercorrelated elementwise with each other and with the criterion (judgment) proximity matrix. Although the independence assumption is violated with this procedure, the obtained correlations provide useful comparative indices of matrix correspondence.

Cluster solutions. Each of the job proximity matrices was next subjected to a cluster analysis, and the resultant cluster solutions were compared with the previously described criterion cluster structure (i.e., the six WTGs). A hierarchical cluster analysis procedure originally described by Ward was employed (Ward, 1961, 1963; Ward \& Hook, 1963). This procedure is included in the Comprehensive Set of Occupational Data Analysis Programs (CODAP) software package developed by the U. S. Air Force (Computer Sciences Corp., 1969; Phalen \& Christal, 1973) and modified for use with the IBM 360 computer system by the U. S. Navy and Marine Corps (Oats-Hill, 1970). The cluster analyses reported here were carried out with a CODAP package that had been further adapted to the IBM $370 / 165$ computer system employed in this study. With appropriate transformations, the CODAP cluster program will accommodate either similarity or dissimilarity measures.

A comparison of a particular cluster analytic solution with the criterion WTG structure involved (1) matching each cluster in the solution with one of the six WTGs and (2) counting the number of misplaced jobs in all clusters vis-á-vis their matched WTGs (i.e., the number of jobs in all clusters that did not belong in their clusters' matched WTGs). Under this procedure, a cluster was matched with the WTG that accounted for the largest number of its constituent jobs. In instances where two clusters in a solution were assigned to the same WTG, the cluster with the fewest jobs from that WTG was left unmatched, and all of its jobs were counted as misplaced. Any isolates in a solution were also counted as misplaced. The total number of mis- placed jobs in a cluster solution served as an index of disparity between that solution and the criterion WTG structure.

\section{Results}

Table 3 presents the correlations among the eight job proximity matrices. As shown in the last column of this table, the matrices based on cosine, correlation, and PO correlated considerably higher with the criterion $(\bar{J})$ matrix than did the matrices based on the remaining profile association measures. The correlations of these three measures with the criterion fell within a narrow range of .700 to .717 , and their correlations with each other ranged from .900 to .979. The correlations of the remaining measures with the criterion ranged from .235 to .368 .

Table 4 presents the results of the comparisons of eight cluster analytic solutions with the criterion WTG structure. As shown, the judgment matrix yielded a solution containing only two misplaced jobs vis-á-vis the WTG structure, whereas the cosine and correlation matrices produced solutions containing seven and eight misplacements, respectively. The number of misplacements in the remaining solutions ranged from 13 with $\mathrm{PO}$ to 25 with $\mathrm{AO}$.

\section{Discussion}

Of the seven proximity measures compared in this study, correlation, cosine, and PO clearly produced the best results in relation to the criterion $(\bar{J})$ proximity matrix. The proximity matrices based on these three measures correlated most highly and about equally with the pairwise similarity judgment matrix. Moreover, these three measures correlated more highly with each other than with the remaining four indices. Interestingly, the $D^{2}$ index, used in the previously cited study by Riccobono et al. (1975), correlated lowest with the criterion matrix.

The same three indices-correlation, cosine, and PO-also outperformed the remaining proximity measures vis-á-vis the criterion 


\section{Table 3 \\ Correlations Among Eight Matrices Containing \\ Different Job Proximity Measures}

\begin{tabular}{llllllll}
\hline $\begin{array}{c}\text { Proximity } \\
\text { Measure }\end{array}$ & $\mathrm{D}^{2}$ & AND & Cos & $\underline{r}$ & PO & AO & $\bar{J}^{\mathrm{a}}$ \\
\hline $\mathrm{D}_{2}$ & .973 & .613 & .534 & .633 & .452 & -.276 & .329 \\
$\mathrm{D}^{2}$ & & .566 & .435 & .531 & .639 & -.294 & .235 \\
$\mathrm{AND}$ & & & .398 & .389 & .365 & .124 & .343 \\
COS & & & & .979 & .954 & .397 & .717 \\
$\mathrm{r}$ & & & & .900 & .248 & .702 \\
PO & & & & & .520 & .700 \\
$\mathrm{AO}$ & & & & & & & .368 \\
\hline
\end{tabular}

\footnotetext{
Note: The column and row designations, with the exception of $\bar{J}$, are defined in Table 2.

$\mathrm{a}_{\mathrm{J}}$ represents the mean pairwise job similarity judgment.
}

(WTG) cluster structure. Against this criterion, however, PO misplaced more jobs than either cosine or correlation, which performed about equally well (with seven and eight misplacements, respectively). AO misplaced the largest number of jobs in this analysis, and the three distance measures were tied for the next largest number of misplacements.

That the pairwise judgment $(\overline{\mathrm{J}})$ matrix produced the best cluster solution, with only two misplacements in relation to the WTG structure, offers some ex post facto support for its use as a criterion in the first comparative analysis. This result suggests that overall similarity judgments of jobs may be more valid than job similarities determined from profile associations. There is the possibility, however, that the judges' pairwise similarity ratings were influenced by some common (though not necessarily valid) conception of job similarity, which also influenced the formation of the WTGs. This shared implicit consensus of job similarity, if it exists, would tend to produce a cluster structure more closely correspondent to the WTGs than would be the case with cluster structures based on profile associations. It should also be noted that the relatively small sample of jobs in this study probably approaches a practical limit for pairwise similarity judgments. In comparison, the numerical profile offers large-sample clustering capability and provides a basis for interpreting a cluster structure after it has been derived.

The results of this study are in general conformance with the predictions. As anticipated, correlation and cosine, the measures least affected by monotonic transformations of score profiles, performed best in relation to the two criteria, whereas the measures most sensitive to such transformations, the distance and $\mathrm{AO}$ indices, performed worst. PO, a measure whose sensitivity to monotonic profile changes falls somewhere between the above-mentioned extremes, also fell between the extremes in performance. Contrary to expectations, removing the influence of paired zero scores from Euclidean distance (with the AND index) did not improve that measure's performance.

It seems likely that interrater differences in the profile data reduced the effectiveness of the distance measures to a considerably greater extent than that of the angular measures. Since different jobs were rated by different analysts, any individual differences in adaptation level and rating dispersion would have been confounded with the true level and scatter com- 


\begin{tabular}{|c|c|}
\hline Proximity measure employed & Number misplaced \\
\hline Euclidean distance & 15 \\
\hline Squared Euclidean distance & 15 \\
\hline Average non-zero distance & 15 \\
\hline Cosine & 7 \\
\hline Product-moment correlation & 8 \\
\hline Percent overlap & 13 \\
\hline Absolute overlap & 25 \\
\hline Mean pairwise job similarity judgment & 2 \\
\hline
\end{tabular}

ponents in the profile data. To the extent that this effect occurred, it could be expected to distort profile association indices that are sensitive to the level and scatter parameters. In such cases, it might prove desirable to exclude these two components from the measure of job proximity.

This study clearly demonstrates the importance of the choice of a profile association measure in cluster analysis. The researcher should be especially cautious when clustering entities that have been rated by different judges. Such situations occur with some frequency and involve a variety of rated stimuli (e.g., jobs, objects, people, and oneself). Under these circumstances, it might be advisable to try out more than one alternative association measure in cluster analyzing a set of profile data. Apropos this suggestion, Skinner (1978) presents a model for differentiating the contributions of elevation, scatter, and shape in profile similarity. Skinner's procedure allows the investigator to "... run several analyses on the same entities, highlighting alternative aspects of profile resemblance" (p. 307). The results of these analyses can then be compared for clarity and stability.

\section{References}

Anderberg, M. R. Cluster analysis for applications. New York: Academic Press, 1973.
Archer, W. B. Computation of group job descriptions from occupational survey data (PRL-TR-66-12). Lackland Air Force Base, TX: Personnel Research Laboratory, Aerospace Medical Division, December 1966. (NTIS No. AD 653 543)

Baker, R. F., \& Young, F. W. Interactive scaling with individual subjects II: An improved system (Report No. 32). Chapel Hill: University of North Carolina, L. L. Thurstone Psychometric Laboratory, April 1974.

Boese, R. R., \& Cunningham, J. W. Systematically derived dimensions of human work. JSAS Catalog of Selected Documents in Psychology, 1976, 6, 57. (Ms. No. 1270)

Borgen, F. H., \& Weiss, D. J. Cluster analysis and counseling research. Journal of Counseling Psychology, 1971, 18, 583-591.

Computer Sciences Corporation. Program description manual-CODAP-computerized occupational data analysis programs. El Segundo, CA: Computer Sciences Corporation, September 1969.

Cronbach, L. J., \& Gleser, G. C. Assessing similarity between profiles. Psychological Bulletin, 1953, 50, 456-473.

Cunningham, J. W., Tuttle, T. C., Floyd, J. R., \& Bates, J. A. The development of the Occupation Analysis Inventory: An "ergometric" approach to an educational problem. JSAS Catalog of Selected Documents in Psychology, 1974, 4, 144. (Ms. No. 803)

Horn, J. L. An empirical comparison of methods for estimating factor scores. Educational and Psychological Measurement, 1965, 25, 313-322.

Morsh, J. E. Identification of job types in the personnel career field (PRL-TR-65-9). Lackland Air Force Base, TX: Personnel Research Laboratory, 
Aerospace Medical Division, April 1965. (NTIS No. AD 622 433)

Nunnally, J. C. Psychometric theory (2nd ed.). New York: McGraw-Hill, 1978.

Oats-Hills Consultants, Inc. CODAP/360: User's guide. Washington, DC: Author, 1970. (prepared for Task Analysis Branch, Headquarters, U. S. Marine Corps).

Osgood, C. E., \& Suci, G. J. A measure of relation determined by both mean differences and profile information. Psychological Bulletin, 1952, 49. 251-262.

Phalen, W. J., \& Christal, R. E. Comprehensive occupational data analysis programs (CODAP). group membership (GRMBRS/GRPMBR), and automated diagramming (DIAGRM) programs (AFHRL-TR-73-5). Lackland Air Force Base, TX: Personnel Research Division, Air Force Human Resources Laboratory, April 1973. (NTIS No. AD 767 199)

Riccobono, J. A., \& Cunningham, J. W. Work dimensions derived through systematic job analysis: A replicated study of the Occupation Analysis Inventory. JSAS Catalog of Selected Documents in Psychology, 1974, 4, 146. (Ms. No. 807)

Riccobono, J. A., Cunningham, J. W., \& Boese, R. R. Clusters of occupations based on systematically derived work dimensions: An exploratory study. JSAS Catalog of Selected Documents in Psychology. 1975, 5, 352. (Ms. No. 1150)

Skinner, H. A. Differentiating the contribution of elevation, scatter, and shape in profile similarity. Educational and Psychological Measurement. 1978, 38, 297-308.

U. S. Department of Labor, Bureau of Employment Security. Dictionary of occupational titles: Occupational classification (Vol. 2, 3rd ed.). Washington, DC: U. S. Government Printing Office, 1965.

Ward, J. H., Jr. Hierarchical grouping to maximize payoff (WADD-TN-61-29). Lackland Air Force
Base, TX: Personnel Research Laboratory, Wright Air Development Division, March 1961. (NTIS No. AD 261 750)

Ward, J. H., Jr. Hierarchical grouping to optimize an objective function. Journal of the American Statistical Association, 1963, 58, 236-244.

Ward, J. H., Jr., \& Hook, M. E. Application of a hierarchical grouping procedure to a problem of grouping profiles. Educational and Psychological Measurement, 1963, 23, 69-76.

Winer, B. J. Statistical principles in experimental design (2nd ed.). New York: McGraw-Hill, 1971.

\section{Acknowledgments}

This article is based on a master's thesis completed at North Carolina State University by the first author under the supervision of the second author. The research was supported by the National Institute of Education. Contract No. NE-C-00-3-0070 (J. W. Cunningham, Principal Investigator). Views expressed in this paper do not necessarily reflect those of the National Institute of Education or any agency of the United States Government.

We thank John J. Pass and Ronald R. Boese for their assistance in the study, Paul W. Thayer and Katherine W. Klein for their helpful comments on an earlier draft of this paper, and Donald H. Mershon for graphical assistance. We also acknowledge the helpful comments and suggestions of an anonymous reviewer.

Robert M. Hamer is now with the Department of Psychiatry, Medical College of Virginia, Richmond, $V A 23298$.

\section{Author's Address}

Send requests for reprints and further information to J. W. Cunningham, Department of Psychology, North Carolina State University, Raleigh, NC 27650. 